\title{
Numerical analysis of vibration modes of a qPlus sensor with a long tip
}

\author{
Kebei Chen ${ }^{\ddagger 1,2,3}$, Zhenghui Liu ${ }^{\ddagger 2,3}$, Yuchen $X_{i e}^{2}$, Chunyu Zhang ${ }^{2,3}$, Gengzhao Xu ${ }^{* 2,3}$, \\ Wentao Song ${ }^{*} 2,3$ and $\mathrm{Ke} \mathrm{Xu}^{2,3}$
}

\section{Full Research Paper}

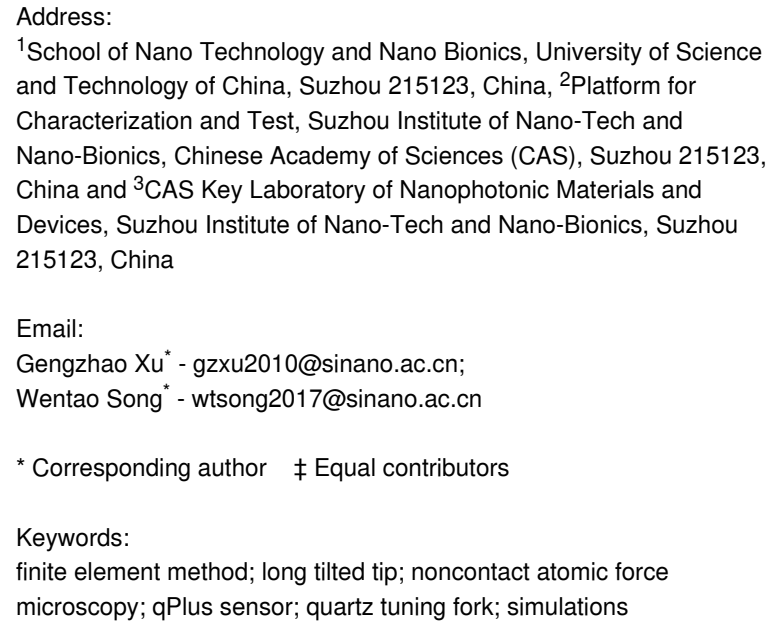

${ }^{1}$ School of Nano Technology and Nano Bionics, University of Science and Technology of China, Suzhou 215123, China, ${ }^{2}$ Platform for Characterization and Test, Suzhou Institute of Nano-Tech and Nano-Bionics, Chinese Academy of Sciences (CAS), Suzhou 215123, China and ${ }^{3}$ CAS Key Laboratory of Nanophotonic Materials and Devices, Suzhou Institute of Nano-Tech and Nano-Bionics, Suzhou 215123, China

Email:

Gengzhao Xu* - gzxu2010@sinano.ac.cn;

Wentao Song* - wtsong2017@sinano.ac.cn

* Corresponding author $\ddagger$ Equal contributors

\section{Keywords:}

finite element method; long tilted tip; noncontact atomic force microscopy; qPlus sensor; quartz tuning fork; simulations

Beilstein J. Nanotechnol. 2021, 12, 82-92. https://doi.org/10.3762/bjnano.12.7

Received: 19 August 2020

Accepted: 20 December 2020

Published: 21 January 2021

Associate Editor: J. Frommer

(C) 2021 Chen et al.; licensee Beilstein-Institut. License and terms: see end of document.

\begin{abstract}
We study the oscillatory behavior of qPlus sensors with a long tilted tip by means of finite element simulations. The vibration modes of a qPlus sensor with a long tip are quite different from those of a cantilever with a short tip. Flexural vibration of the tungsten tip will occur. The tip can no longer be considered as a rigid body that moves with the prong of the tuning fork. Instead, it oscillates both horizontally and vertically. The vibration characteristics of qPlus sensors with different tip sizes were studied. An optimized tip size was derived from obtained values of tip amplitude, ratio between vertical and lateral amplitude components, output current, and quality factor. For high spatial resolution the optimal diameter was found to be $0.1 \mathrm{~mm}$.
\end{abstract}

\section{Introduction}

Quartz tuning forks are widely used in the watch industry because of their low frequency offset over a wide temperature range [1]. In addition, quartz tuning forks have a high elastic constant, a high quality factor ( $Q$ factor), and are self-sensing due to the piezoelectric effect [1]. Therefore, a quartz tuning fork can be used as a force sensor. The central part of the "qPlus sensor" is a quartz tuning fork of which one prong is fixed onto a substrate and the other prong with an attached tip serves as a self-sensing cantilever [2]. In 1996, F. J. Giessibl et al. first used the qPlus sensor to measure the morphology of a grating 
and a CD at room temperature [3]. Since then, this technique has been used extensively in the fields of physics, chemistry, and materials science to obtain images of high spatial resolution [4-12].

Most research under low-temperature and ultrahigh-vacuum (LT-UHV) conditions has been carried out using the first-order eigenmode of the qPlus sensor, which has a short tip that can be considered as a rigid body vertically attached to the tuning fork prong. However, soft biological samples, such as living cells and lipid membranes [13-15], must be immersed in a liquid environment to maintain their original properties. In order to avoid immersion of the tuning fork and the wiring in the liquid, a longer tip is required. By keeping the nodes of the tip in the higher-order modes close to the liquid surface, the frequency drift of the sensor can be effectively limited, maintaining a high $Q$ factor [16,17]. By using a qPlus sensor with a long tip, atomic resolution and near-field optical images were obtained in liquid environments $[15,18,19]$.

For some applications, it is required that the tip is not perpendicular to the prong of the tuning fork [20,21]. The advantage of a qPlus sensor with a long tilted tip (Figure 1) is that forces in multiple directions can be detected due to the multi-directional vibration of the tip [17]. Furthermore, by using a qPlus sensor with a long tilted tip, vertical incident light can be coupled to the tip apex. This setup has the added benefit of locating the exact target location with high resolution when it is combined with an optical microscope to observe the sample surface from upside $[22,23]$. However, there is only scarce work regarding the analysis of how the shape of the long tilted tip affects the vibration of the sensor. The selection of tip length and diameter often depends on the experience of researchers.

Here, we report a numerical study of the vibration modes of qPlus sensors. Eigenfrequencies, tip amplitudes, ratios between vertical and lateral amplitude components, output currents, and $Q$ factor values as functions of the tip size of the qPlus sensor are systematically analyzed. Eventually, the optimal tip size can be derived from the simulation results.

\section{Methods \\ Model and parameters}

The model in the simulation is based on an MS1V-T1K-type quartz tuning fork used in our experiment (details will be described later). The dimensions of the MS1V-T1K quartz tuning fork, length $L=3423 \mu \mathrm{m}$, width $W=687 \mu \mathrm{m}$, and thickness $\tau=121 \mu \mathrm{m}$, are used in the finite element model. The arrangement of the gold electrodes on the tuning fork is the same as in reality. One electrode pair is grounded and the other is set to a virtual ground. The calculated mechanical eigenfre-

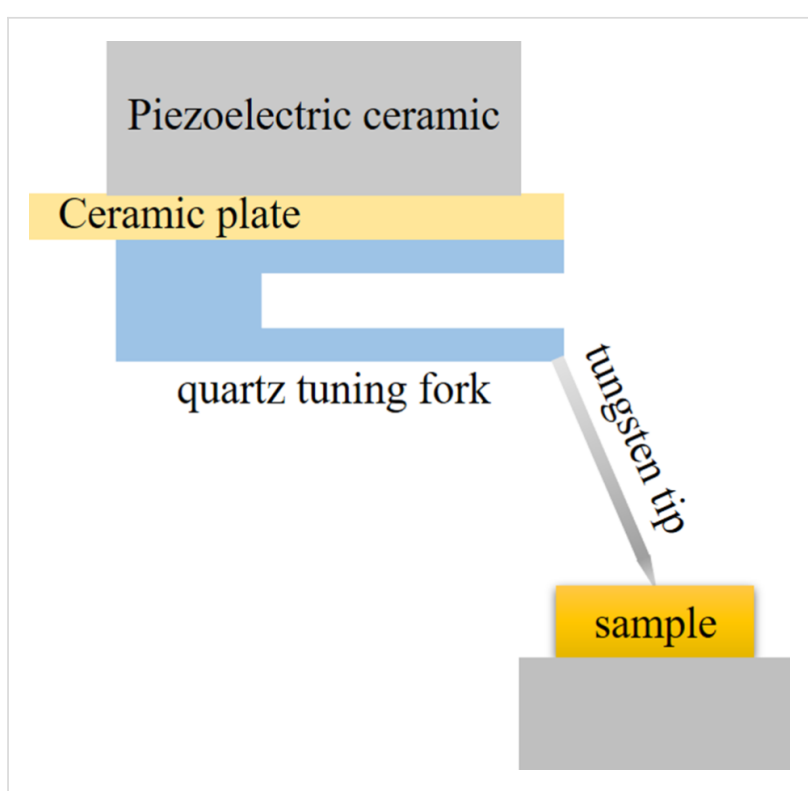

Figure 1: Schematic diagram of a qPlus sensor with a long tilted tip.

quency of the bare tuning fork is $32.19 \mathrm{kHz}$. After considering the electrostatic and piezoelectric effects, the eigenfrequency is shifted to $32.23 \mathrm{kHz}$, which is only $0.12 \%$ higher. Therefore, the effect of the shunt capacitance of the tuning fork is negligible.

As shown in Figure 2a, the tungsten tip is attached to one prong using a rectangular drop of Torr seal epoxy. The diameter values of the tungsten tip used in the simulations were chosen according to diameters of the tungsten wire available [24]. We selected four different diameters: $0.025 \mathrm{~mm}, 0.05 \mathrm{~mm}$, $0.075 \mathrm{~mm}$, and $0.1 \mathrm{~mm}$. The tip length can be customized according to the experimental needs. In this paper, nine lengths are selected for calculation at each diameter: $0.5 \mathrm{~mm}, 0.65 \mathrm{~mm}$, $0.8 \mathrm{~mm}, 1.0 \mathrm{~mm}, 1.2 \mathrm{~mm}, 1.4 \mathrm{~mm}, 1.5 \mathrm{~mm}, 1.7 \mathrm{~mm}$, and $2 \mathrm{~mm}$. The angle between the tip and the prong is $65^{\circ}$. An external excitation is applied by exerting a harmonic displacement of $4.5 \mathrm{~nm}$ on the side of the tuning fork prong without a tip. Figure $2 \mathrm{~b}$ visualizes the mesh distribution used for the calculations. The mesh density increases at the link between the two prongs and near the boundaries of each material. Figure $2 \mathrm{c}$ is a zoomed view of the tungsten wire attached to the end of the tuning fork. The epoxy glue is defined as a cuboid in order to facilitate mesh generation and reduce calculation time, since it is not guaranteed to have a fixed geometry after curing. The glue thickness in the simulation is set to be the average glue thickness of the qPlus sensor used in our experiment. In this case, the volume of the glue in the simulation is approximately equal to the volume of the glue in the experiment. Figure $2 d$ is a false-color representation of the total displacement levels. Warmer colors represent a larger displacement. 
(a)
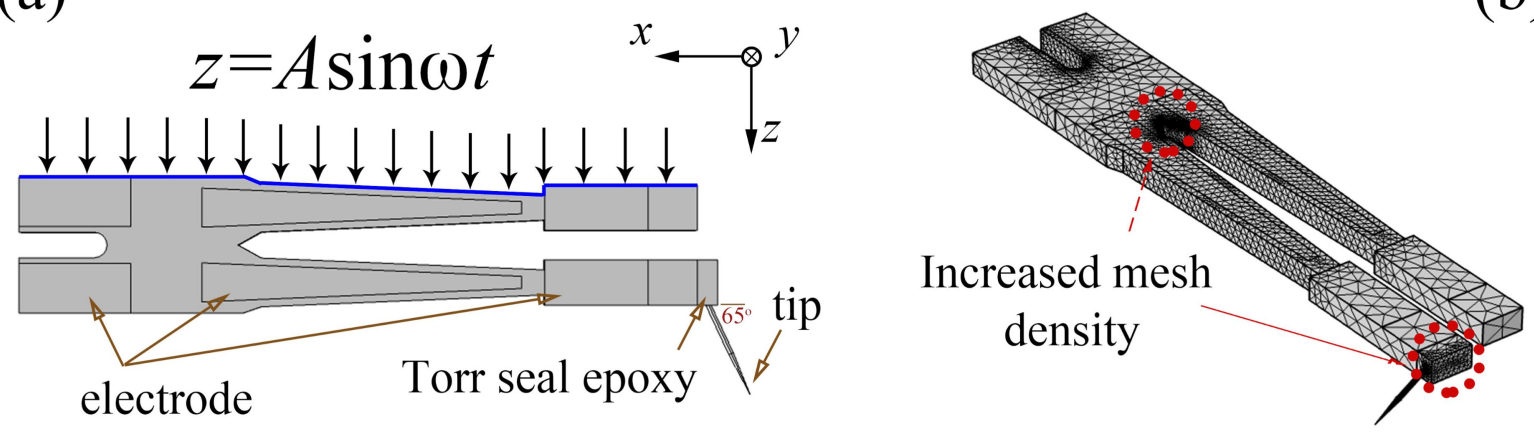

(c)

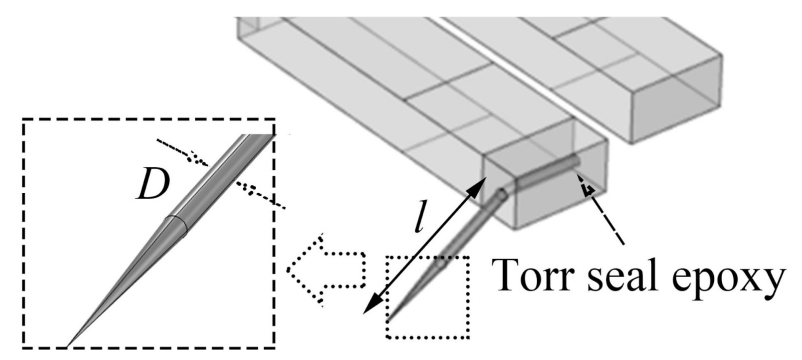

(d)

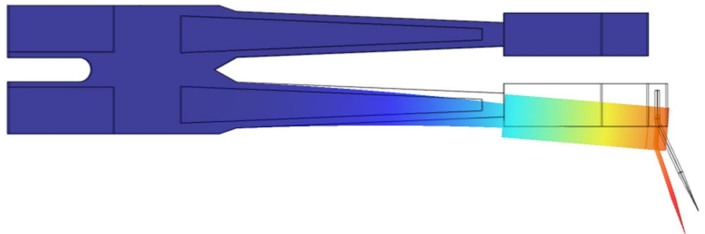

Figure 2: (a) Schematic diagram of the simulation model including tuning fork, Torr seal epoxy glue, and tungsten tip. Mechanical vibration excitation is applied on one side of the tuning fork. (b) Map of the mesh distribution as implemented in the finite element simulation. (c) Zoomed-in view of the tungsten wire attached to the end of the tuning fork. (d) False-color representation of the total displacement levels when the free prong deforms under excitation.

All calculations were carried out using COMSOL Multiphysics. Table 1 summarizes the parameters used, including Young's modulus, Poisson's ratio, mass density, and damping coefficients for all materials considered. The values for Torr seal epoxy were chosen as in the papers by Dennis van Vörden et al. [25] and Omur E. Dagdeviren and co-workers [26]. The parameters for quartz, gold, and tungsten were taken from the materials library of the simulation software, except for the damping coefficient for quartz, which was chosen based on our experimental results. According to [26], it is also worth noting that (i) due to the comparatively low internal damping occurring inside gold and tungsten, we did not assign a damping coefficient to any of these materials to reduce the computational cost, and that (ii) the sensor is oscillating in vacuum.

\section{Observation by scanning electron microscope (SEM)}

Firstly, in this paper, the whole assembly consisting of tuning fork, tip, and Torr seal epoxy is referred to as the qPlus sensor. The oscillation of the qPlus sensor with a long tilted tip was experimentally observed using a commercial SEM (FEI Quanta FEG 250). The tungsten wire (Alfa Aesar company) used in the experiment had a diameter of $0.025 \mathrm{~mm}(0.001 \mathrm{in})$ and a purity of $99.95 \%$. The tip was obtained by AC electrochemical etching in $\mathrm{NaOH}$ solution at a concentration of $1 \mathrm{~mol} \cdot \mathrm{L}^{-1}$. A tungsten wire with a length of $942 \mu \mathrm{m}$ was attached to the end of the tuning fork with Torr seal epoxy. The angle between the tungsten wire and the prong was $65^{\circ}$. The excitation signal was introduced via mechanical excitation of the base part by a piezo

Table 1: Material parameters used for finite element calculations.

\begin{tabular}{|c|c|c|c|c|}
\hline Material constant & Quartz & Torr seal epoxy & Gold & Tungsten \\
\hline Young's modulus (GPa) & 78.31 & 9.39 & 70 & 360 \\
\hline Poisson's ratio & 0.14 & 0.45 & 0.44 & 0.28 \\
\hline mass density $\left(\mathrm{kg} \cdot \mathrm{m}^{-3}\right)$ & 2651 & 1600 & 19300 & 19300 \\
\hline damping coefficient & $1 \times 10^{-4}$ & $5 \times 10^{-3}$ & - & - \\
\hline
\end{tabular}


actuator. The excitation amplitude was $1 \mathrm{~V}$. The scanning rate of SEM was $20 \mu \mathrm{s} /$ pixel. The vibration of the qPlus sensor was observed at room temperature while maintaining a pressure of $10^{-3} \mathrm{~Pa}$.

\section{Results and Discussion Eigenfrequencies and eigenmodes}

The eigenfrequencies of three parts are discussed in this paper. These parts are the qPlus sensor, the prong (the free prong of the tuning fork) with one end fixed and the other end carrying a concentrated load which equals the tip mass, and the independent tungsten tip. These three eigenfrequencies are denoted by $f_{\mathrm{q}}, f_{\mathrm{tf}}$, and $f_{\text {tip }}$, respectively. The values of $f_{\mathrm{q}}$ and $f_{\text {tip }}$ are obtained by the simulation, and $f_{\mathrm{tf}}$ is calculated by the method described in [27]. Since the oscillation of the tip is mainly in the tapping mode during scanning, we focus on the modes of the tuning fork prong and the tip oscillating in the $X-Z$ plane. We found that when the tuning fork oscillates at the first-order eigenfrequency, there are two different vibration eigenmodes of the tip, as shown in Figure 3a. We name the vibration mode in which the tip and the tuning fork are deflected in the same direction the "in-phase" mode. If the tip and the tuning fork are deflected in opposite directions, we name the corresponding vibration mode the "anti-phase" mode. Figure 3b-e summarizes the relations between $f_{\mathrm{q}}$ and the tip length for the four different tip diameters. In the in-phase mode, the qPlus sensor oscillates at a lower eigenfrequency, and in the anti-phase mode at a higher eigenfrequency. With the increase of tip diameter, the tuning fork shows the vibration of the second-order eigenmode in the anti-phase mode, especially with the shorter tip. This paper does not discuss the second-order eigenmode of the tuning fork, so there are no corresponding frequencies of the anti-phase mode at some tip lengths in Figure 3c-e.

The vibration of the qPlus sensor is the superposition of the oscillation of the tuning fork and the oscillation of the tip. The relations between $f_{\mathrm{q}}, f_{\mathrm{tf}}$, and $f_{\text {tip }}$ for the qPlus sensor with a diameter of $0.025 \mathrm{~mm}$ tip are shown in Figure 4. The grey dashed lines denote $f_{\mathrm{tf}}$. When the tip length is $0.5 \mathrm{~mm}, f_{\mathrm{q}}$ of the in-phase mode is very close to the dashed line (Figure 4a), which means that the tuning fork vibrates in resonance. When $f_{\mathrm{q}}$ moves away from the dashed line and gets closer to $f_{\text {tip }}$, the vibration of the qPlus sensor is mainly dominated by the tip, while the tuning fork hardly oscillates. The value of $f_{\mathrm{q}}$ of the anti-phase mode is close to that of $f_{\text {tip }}$ for a $0.5 \mathrm{~mm}$ long tip (Figure $4 \mathrm{~b}$ ), resulting in tip resonance. As the tip length increases, $f_{\mathrm{q}}$ gradually approaches $f_{\mathrm{tf}}$, leading to resonance of the tuning fork. If the tip length continues to increase, $f_{\mathrm{q}}$ will be closer to the second-order $f_{\text {tip }}$. The superposition vibration characteristics of the qPlus sensor are reflected in the amplitudes of the tuning fork and the tip, which will be discussed later.

\section{Amplitude of the tuning fork $\left(A_{\mathrm{tfz}}\right)$ and its output current}

In this paper, the amplitude in the $Z$ direction of point $A$ on the tuning fork prong in Figure $3 \mathrm{a}$ is denoted as $A_{\mathrm{tfz}}$. The amplitude of point B at the tip in Figure $3 \mathrm{a}$ is denoted as $A_{\text {tip. }} A_{z}$ and $A_{x}$ represent the two components of the amplitude of the tip apex in the $Z$ and $X$ directions, respectively. $A_{\text {tip }}$ is equal to $\sqrt{A_{x}^{2}+A_{z}^{2}}$. The angle between $A_{\text {tip }}$ and $A_{x}$ is $\varphi$. The frequencies near $f_{\mathrm{q}}$ are scanned by the frequency domain study in COMSOL to obtain the maximum values of $A_{\mathrm{tfz}}, A_{x}, A_{z}$, and the output currents. Figure 5 shows the $A_{\mathrm{tfz}}$ and the simulated output currents.

Rise and drop of $A_{\mathrm{tfz}}$ of both modes in Figure 5a-d can be explained by the tuning fork resonance. We take the $0.025 \mathrm{~mm}$ tip as an example. When the tip length is $0.5 \mathrm{~mm}$, the tuning fork vibrates resonantly in the in-phase mode (Figure 4a), corresponding to the larger $A_{\mathrm{tfz}}$ in Figure 5a. The value of $A_{\mathrm{tfz}}$ decreases due to $f_{\mathrm{q}}$ moving far away from $f_{\mathrm{tf}}$. The value of $A_{\mathrm{tfz}}$ of the anti-phase mode for a $0.5 \mathrm{~mm}$ long tip is small because of the large difference between $f_{\mathrm{q}}$ and $f_{\mathrm{tf}}$ (Figure $4 \mathrm{~b}$ ). With the increase of the tip length, $f_{\mathrm{q}}$ gradually gets closer to $f_{\mathrm{tf}}$, and $A_{\mathrm{tfz}}$ gradually increases as well. The value of $A_{\mathrm{tfz}}$ reaches the maximum value when the tip length is $1.4 \mathrm{~mm}$. At larger tip lengths, $f_{\mathrm{q}}$ gradually moves away from $f_{\mathrm{tf}}$, while $A_{\mathrm{tfz}}$ decreases.

Rise and drop of $A_{\mathrm{tfz}}$ in both modes are also reflected in the simulated output current. Similar to $A_{\mathrm{tfz}}$, the output current with a shorter tip is larger in the in-phase mode, and the output current with a longer tip is larger in the anti-phase mode (Figure 5e-h). However, we found that the intersection points of the output currents do not coincide with those of $A_{\mathrm{tfz}}$ (except for the $0.025 \mathrm{~mm}$ tip). For example, when the output current generated in the anti-phase mode is larger than that in the in-phase mode (Figure $5 \mathrm{~g}$ ), the value of $A_{\mathrm{tfz}}$ of the anti-phase mode is lower than that of the in-phase mode (Figure $5 \mathrm{c}$ ). This is because the frequency of the anti-phase mode is higher, that is, the period is shorter. Although the peak value of the charge $q$ of the anti-phase mode, caused by the piezoelectric effect of the tuning fork [1], is smaller than that of the in-phase mode, the maximum value of the partial derivative of $q$ with respect to the time (i.e., the peak output current) can be larger than that of the in-phase mode. The result illustrates that the changes of the output current and of $A_{\mathrm{tfz}}$ with respect to the tip length are not necessarily synchronous.

\section{Tip oscillation and SEM observation}

Since the tip swings around the end of the tuning fork prong, $A_{\text {tip }}$ is different from $A_{\mathrm{tfz}}$. We should focus on the vibration of the tip apex itself. Figure 6 gives $A_{\text {tip }}$ and the ratio between $A_{x}$ and $A_{z}$ for the four tip diameters with different 

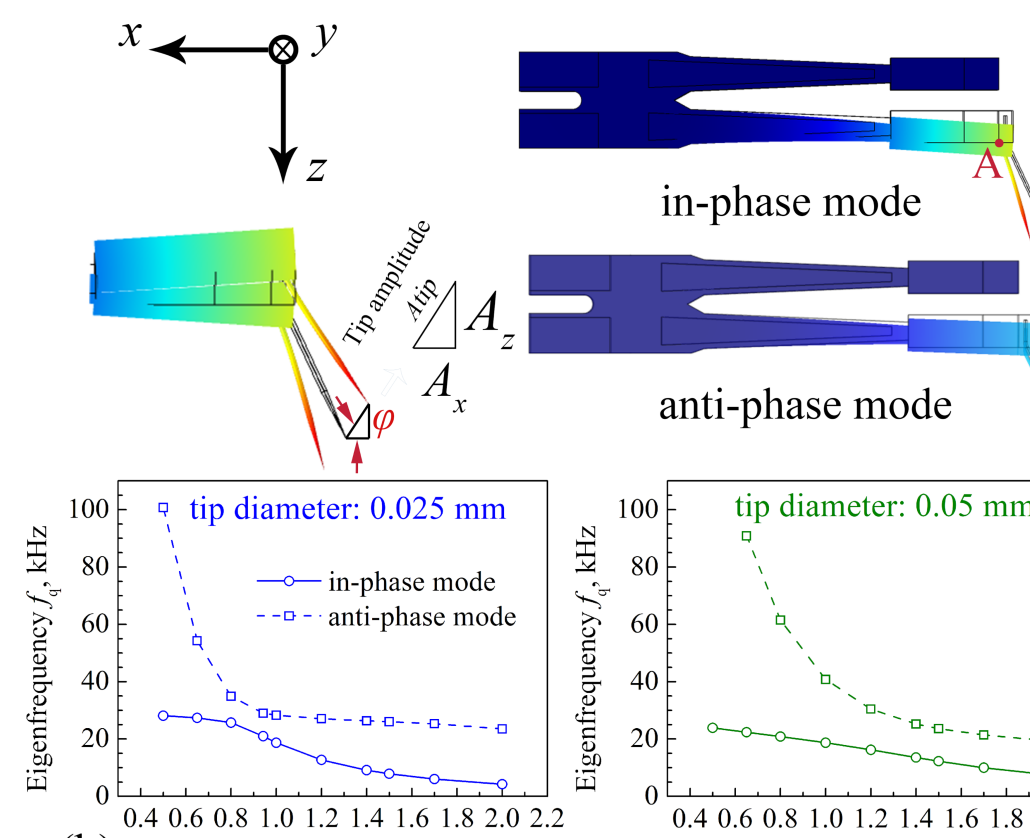

(a)

(b)

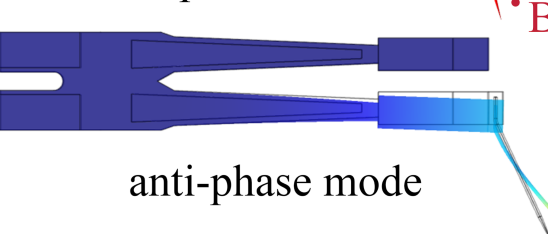
B
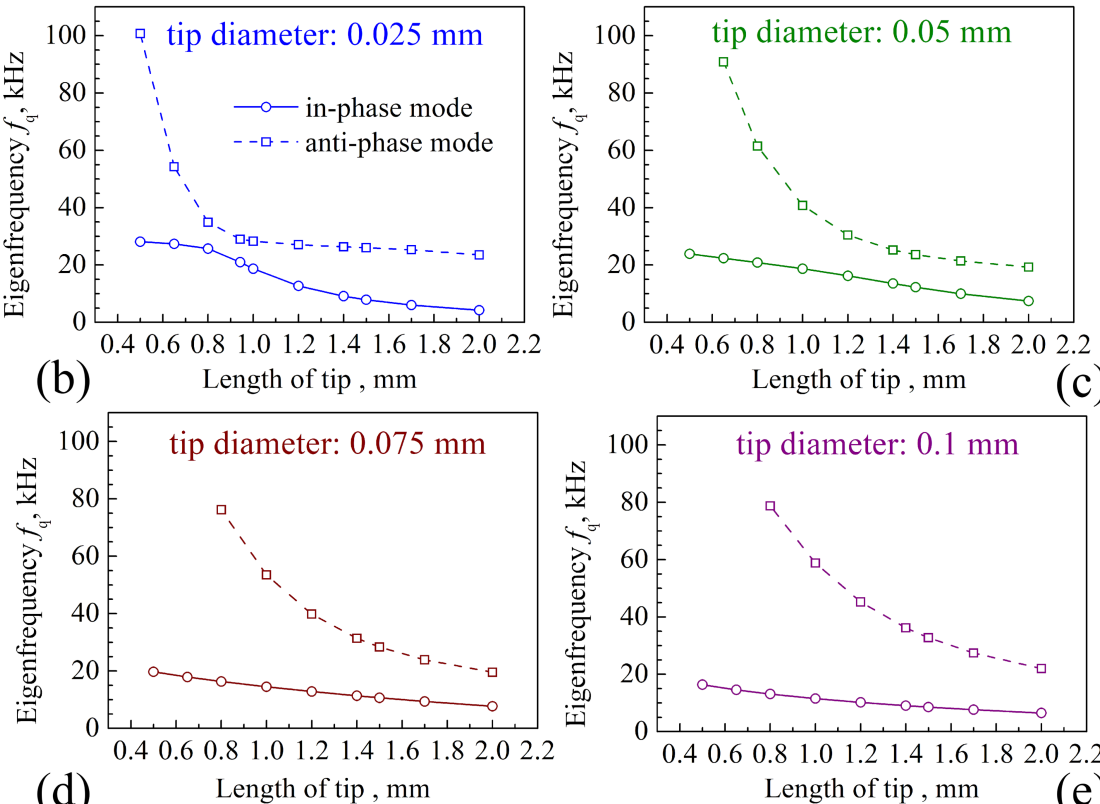

Length of tip, mm

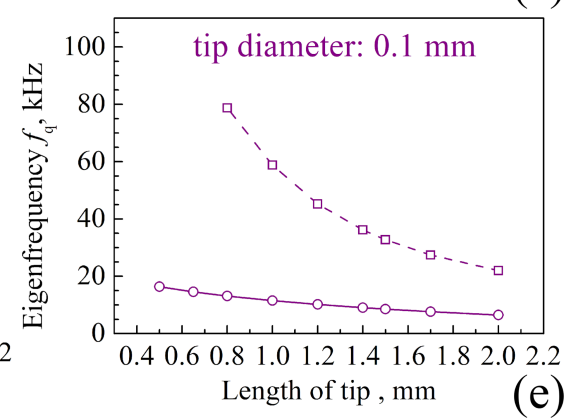

Figure 3: (a) Two different eigenmodes in the simulation. In-phase mode: the tip and the tuning fork deflect in the same direction. Anti-phase mode: the tip and the tuning fork are deflected in the opposite direction. (b-e) The values of $f_{\mathrm{q}}$ of the four tip diameters with different lengths in both eigenmodes.
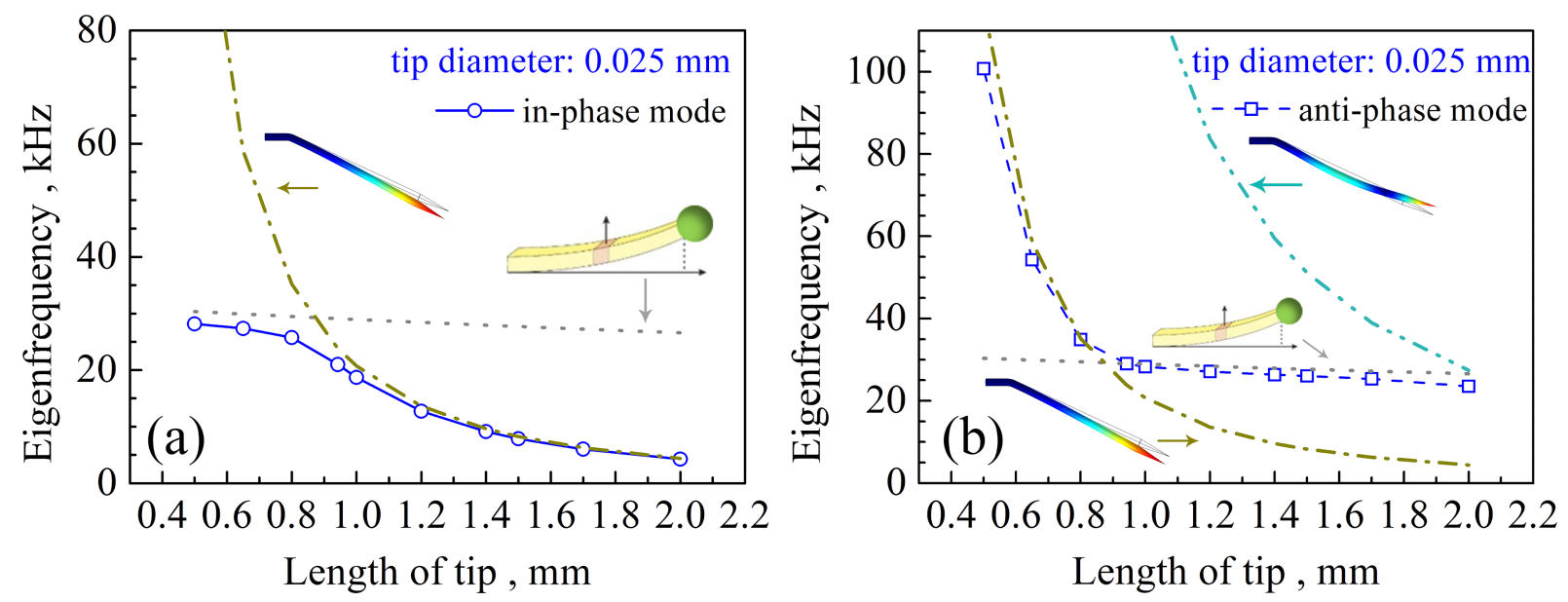

Figure 4: The relations between $f_{\mathrm{q}}, f_{\mathrm{tf}}$, and $f_{\mathrm{tip}}$ for the qPlus sensor with a tip diameter of $0.025 \mathrm{~mm}$ in the in-phase mode (a) and in the anti-mode (b). The eigenmodes corresponding to $f_{\mathrm{tf}}$ and $f_{\mathrm{tip}}$ are shown in the illustrations. 


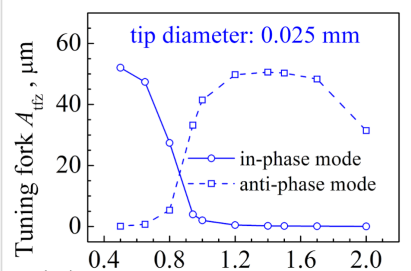

(a)

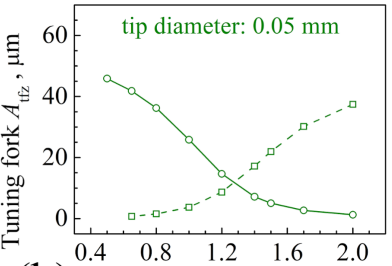

(b)

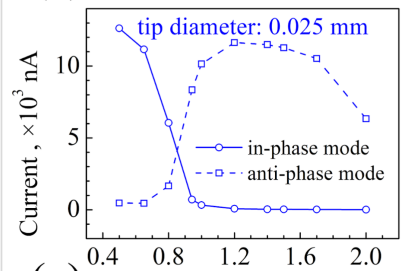

(e) $\begin{array}{cccc}0.4 & 0.8 & 1.2 & 1.6 \\ & \text { Length of tip , mm }\end{array}$

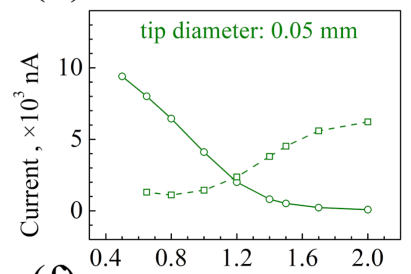

(f) $\begin{array}{cccc}0.4 & 0.8 & 1.2 & 1.6 \\ & \text { Length of tip }, \mathrm{mm}\end{array}$

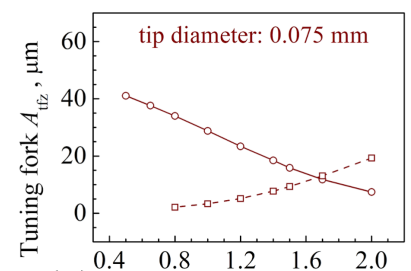

(c) Length of tip, $\mathrm{mm}$

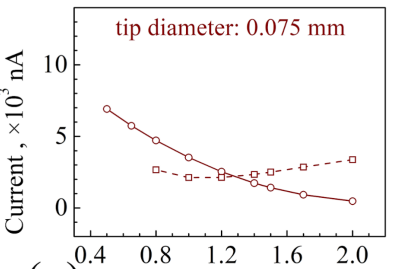

$(\mathrm{g})^{0.4} \quad \begin{array}{lll}0.8 & 1.2 & 1.6 \\ & \text { Length of tip , mm }\end{array}$

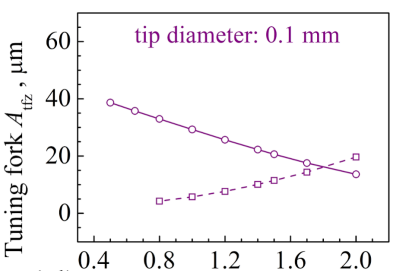

(d) $\quad$ Length of tip , mm

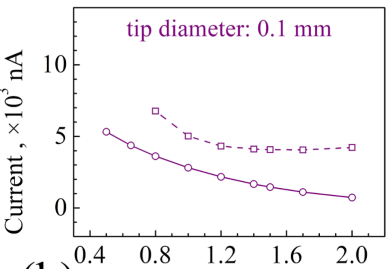

(h) Length of tip , mm

Figure 5: Illustrations of $A_{\mathrm{tfz}}(\mathrm{a}-\mathrm{d})$ and output currents $(\mathrm{e}-\mathrm{h})$ as functions of the tip length for the four different tip diameters.

lengths. We found that for a fixed tip diameter and tip length, $A_{\text {tip }}$ is always greater than $A_{\mathrm{tfz}}$. The value of $A_{x} / A_{z}$ is the cotangent value of the angle $\varphi$ (Figure 3a). If $0<\varphi<45^{\circ}$ then $A_{x} / A_{z}>1$; if $45^{\circ}<\varphi<90^{\circ}$ then $A_{x} / A_{z}<1$. A higher ratio corresponds to a higher proportion of the oscillation parallel to the $X$ direction. In contrast, a smaller ratio means the tip oscillation is close to the ideal tapping mode. In the in-phase mode, $A_{x} / A_{z}$ for all tip diameters increases with respect to the tip length. However, the increase of $A_{x} / A_{z}$ is smaller for $0.075 \mathrm{~mm}$ and $0.1 \mathrm{~mm}$ tips. When the tip gets thinner, the vibration tends to be parallel to the $X$ direction. When the tip gets thicker, the oscillation behaves more like the tapping mode.

The value of $A_{\text {tip }}$ is determined through the vibrations of the tuning fork prong and the tip. Therefore, the change of $A_{\text {tip }}$ cannot be simply analyzed by the method for analyzing $A_{\mathrm{tfz}}$. Again, we take the $0.025 \mathrm{~mm}$ tip as an example. The value of $f_{\text {tip }}$ of the in-phase mode gets closer to $f_{\mathrm{q}}$ with the increase of the tip length (Figure 4a). Accordingly, the value of $A_{\text {tip }}$ shows a constant rise when the tip length varies between 0.5 and $0.8 \mathrm{~mm}$ (Figure $6 \mathrm{a}$ ). When the tip length exceeds $0.8 \mathrm{~mm}, A_{\mathrm{tfz}}$ is greatly reduced to almost zero (Figure $5 \mathrm{a}$ ), which means that the tuning fork hardly oscillates. Therefore, $A_{\text {tip }}$ is also reduced. In the anti-phase mode, the qPlus sensor vibrates near the firstorder $f_{\text {tip }}$ when the tip length ranges between 0.5 and $0.8 \mathrm{~mm}$ (Figure 4b). A longer tip length leads to a larger $A_{\text {tip }}$ (Figure 6a). At the length of $0.942 \mathrm{~mm}$, the tip apex vibrates almost parallel to the $X$ direction. Therefore, $A_{x} / A_{z}$ increases greatly (Figure 6e). However, $A_{\text {tip }}$ begins to decrease when the tip is longer than $0.942 \mathrm{~mm}$. This is caused by the change of $A_{\mathrm{tfz}}$ and the relative position of the node on the tip. The deflection directions of the tuning fork and the tip are opposite in the anti-phase mode. When the tip is longer than $0.8 \mathrm{~mm}, A_{\mathrm{tfz}}$ increases quickly with respect to the tip length and the node of the tip moves towards the tip apex. Therefore, $A_{\text {tip }}$ decreases. At a tip length of $1.4 \mathrm{~mm}, A_{\text {tip }}$ reaches its minimal value. Because $f_{\mathrm{q}}$ of the anti-phase mode gets much closer to the second-order $f_{\text {tip }}$ when the tip length is longer than $1.4 \mathrm{~mm}, A_{\text {tip }}$ increases at the same time, and the tip apex oscillates parallel to the $X$ direction again, resulting in a corresponding increase of $A_{x} / A_{z}$.

In order to understand the oscillation behavior of the tip more intuitively, a qPlus sensor with a tip length of $942 \mu \mathrm{m}$ was fabricated and placed in an SEM to observe the vibration, as shown in Figure 7a. We carried out a series of frequency sweep experiments from 18 to $32 \mathrm{kHz}$ in ambient atmosphere. The obtained response curve is plotted in Figure $7 \mathrm{~b}$. We found resonance frequencies of 20.64 and $28.51 \mathrm{kHz}$ in the scanning curve, which are close to the frequencies of $20.98 \mathrm{kHz}$ of the in-phase mode and $28.99 \mathrm{kHz}$ of the anti-phase mode, respectively, from the simulation. When we used a driving frequency between 28.62 and $28.78 \mathrm{kHz}$ in the SEM, the resonance frequency of the qPlus sensor was found at $28.71 \mathrm{kHz}$ (Figure 7d), which is slightly higher than that measured in atmosphere due to less air damping. The oscillation of the apex of the tip is presented in the Figure 7c. The value of $A_{\text {tip }}$ measured directly from the SEM images was $1.435 \mu \mathrm{m}, A_{z}$ and $A_{x}$ were 0.47 and $1.35 \mu \mathrm{m}$, respectively. The ratio between $A_{x}$ and $A_{z}$ was 2.85 . In the simulation the values were $A_{z}=18.09 \mu \mathrm{m}, A_{x}=110.81 \mu \mathrm{m}$, and $A_{x} / A_{z}=6.12$. One possible reason for the lower value of $A_{x} / A_{z}$ in the SEM is the expansion of the epoxy glue from the tuning fork to the tip (Figure 7a). This is confirmed by a further simulation (see Supporting Information File 1). 

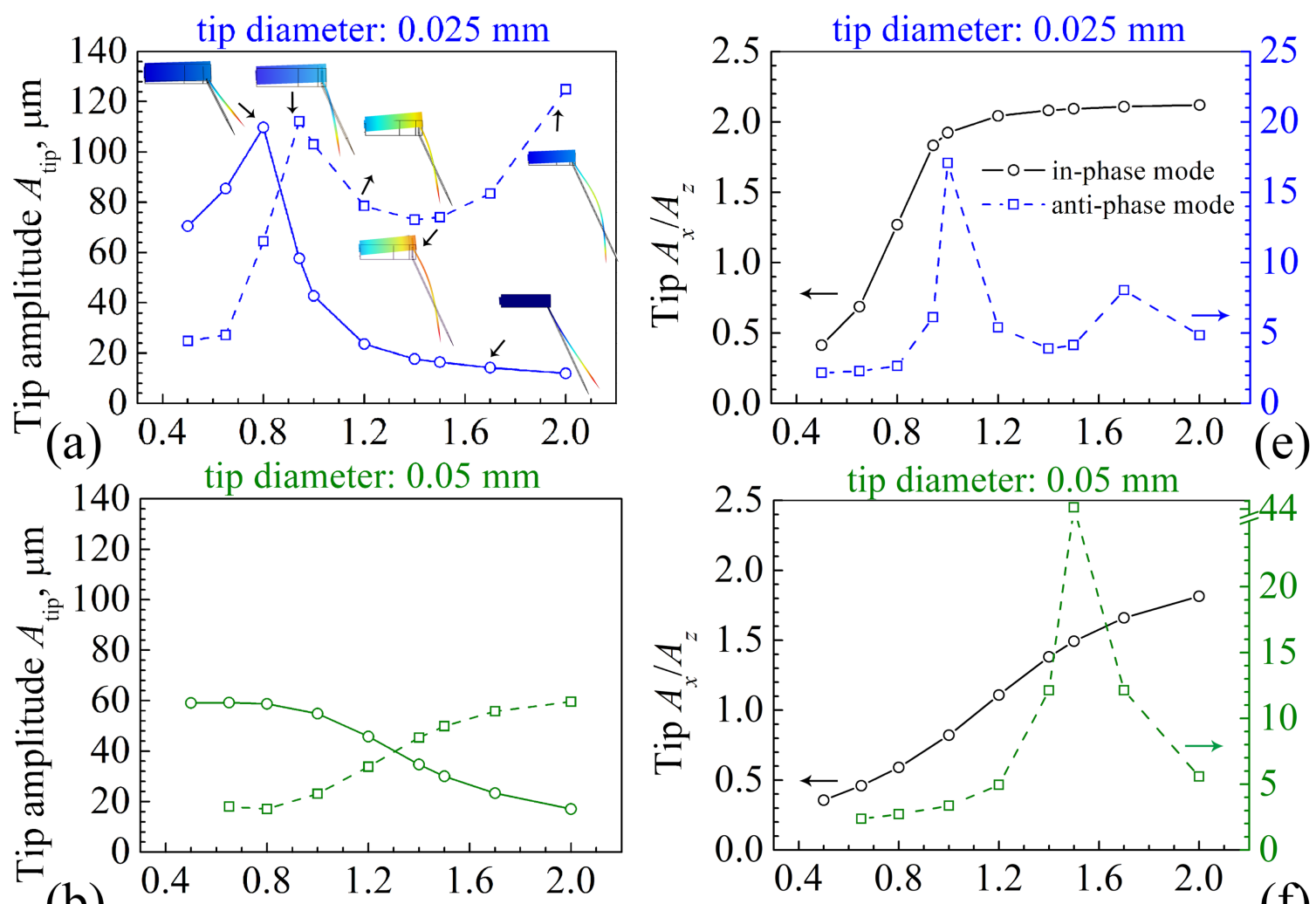

(b) tip diameter: $0.075 \mathrm{~mm}$
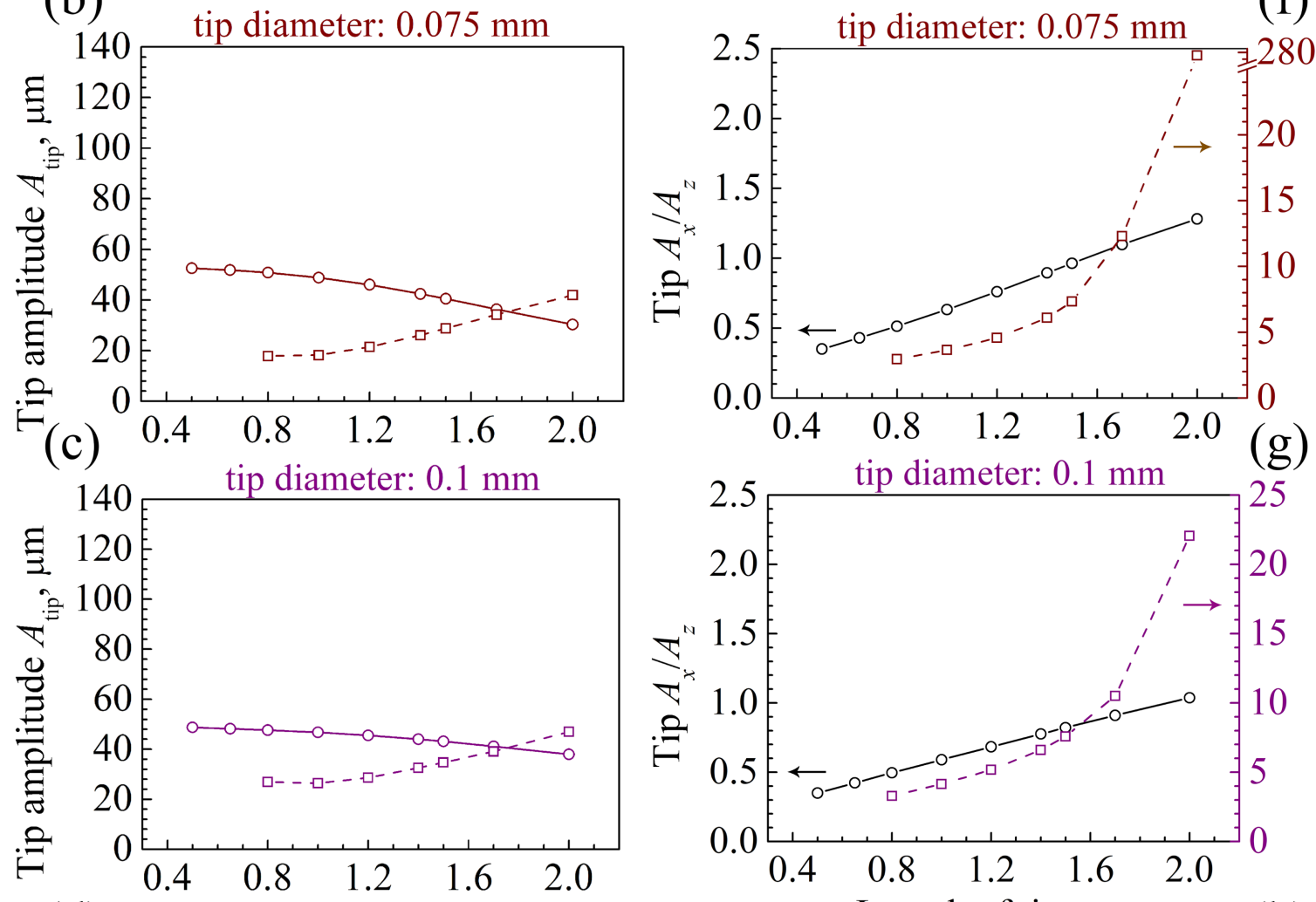

(d) Length of tip , $\mathrm{mm}$

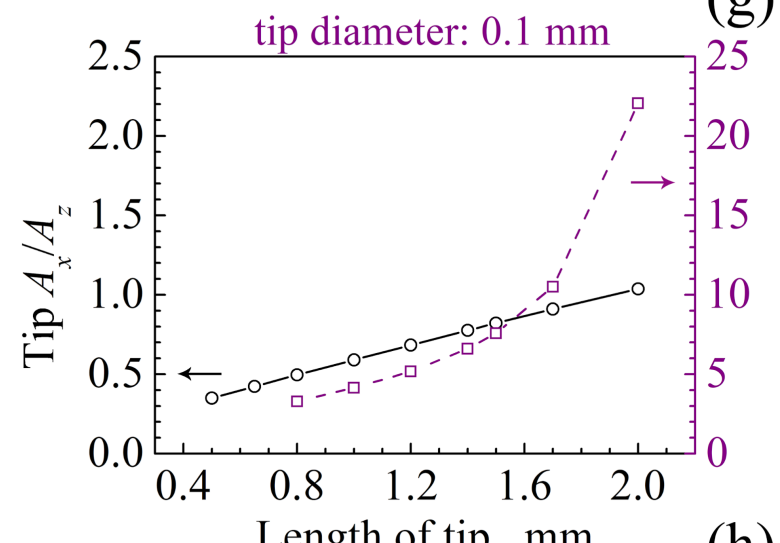

Figure 6: (a-d) $A_{\text {tip }}$ and (e-h) $A_{x} / A_{z}$ as functions of the tip length depicted for the four different tip diameters. The illustrations in (a) show the oscillation behaviors of the $0.025 \mathrm{~mm}$ tip with different lengths in both modes. 

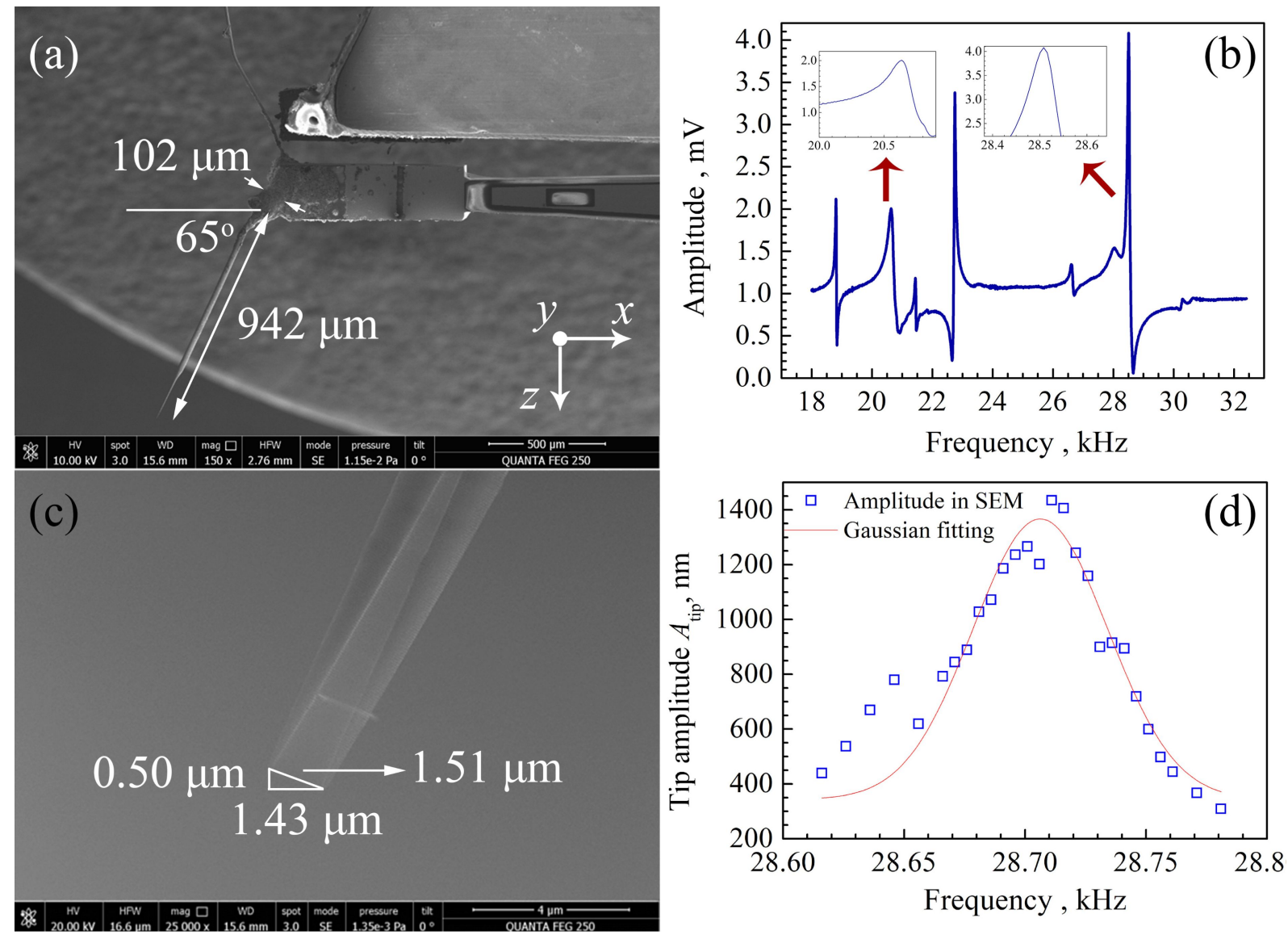

Figure 7: (a) SEM observation of a qPlus sensor with an attached tungsten tip with a length of about $0.942 \mathrm{~mm}$ and a diameter of $0.025 \mathrm{~mm}$. (b) Sweeping frequency curve in ambient atmosphere. 20.64 and $28.51 \mathrm{kHz}$ correspond to the values of $f_{\mathrm{q}}$ of the in-phase mode and the anti-phase mode, respectively, from the simulation. (c) Corresponding SEM images taken around the oscillating tip apex. The value of $A_{\text {tip }}$ is $1.51 \mu$ m, and $A_{x}$ and $A_{z}$ are 1.43 and $0.50 \mu \mathrm{m}$, respectively. (d) Blue squares: $A_{\text {tip }}$ measured from SEM images. Red line: fit with a Gaussian curve. The resonance frequency of $28.71 \mathrm{kHz}$ of the anti-phase mode measured in SEM is slightly higher than that measured in atmosphere due to less air damping.

\section{Tip selection}

The purpose of optimizing the tip dimensions is to improve the spatial resolution of the measurement. Therefore, we need to consider all physical parameters comprehensively. Firstly, it is necessary to reduce the influence of the long-range force, where $A_{\text {tip }}$ should be small enough. Secondly, $f_{\mathrm{q}}$ should be high to minimize frequency noise [1]. However, there is a tradeoff between $A_{\text {tip }}$ and $f_{\mathrm{q}}$ in the in-phase mode (Figure 3 and Figure 6). We found a $0.05 \mathrm{~mm}$ tip has the best performance when the tip length is $0.65 \mathrm{~mm}$ in the anti-phase mode. However, $A_{x} / A_{z}$ in the anti-phase mode is 2.36 , that is, $\varphi$ is $23^{\circ}$. In frequency modulation-atomic force microscopy (FM-AFM), the frequency shift $\Delta f$ of the cantilever is utilized to detect the forces between tip and sample. The shift $\Delta f$ induced by the vertical force gradient is $\tan |\varphi|$ times as large as that induced by the lateral force gradient [17]. In other words, in the range from 0 to $90^{\circ}$, if $\varphi>45^{\circ}$, mainly the vertical force gradient contributes to $\Delta f$. If $\varphi<45^{\circ}$, the lateral force gradient has a greater impact. For most cases, it is desirable to detect a larger vertical force gradient signal, so we need $A_{X} / A_{z}<1$. The in-phase modes were found to fulfil this requirement. Thirdly, we want the stiffness of the qPlus sensor to be large enough to allow for a stable smallamplitude operation [1]. The equivalent stiffness $k_{\text {eq }}$ of the qPlus sensor is shown in Figure 8, which is calculated from the strain energy and the tip amplitude with an equivalent pointmass model [28]. We see in Figure 8c that for a $0.075 \mathrm{~mm}$ tip, there is an optimal tip length of $2 \mathrm{~mm}$ that will achieve the maximum $k_{\text {eq }}$. However, the corresponding vibration is in the anti-phase mode. In the in-phase mode, $k_{\text {eq }}$ decreases with the increase of the tip length. When the tip length is longer than $0.65 \mathrm{~mm}$, the value of $k_{\text {eq }}$ of the qPlus sensor with $0.1 \mathrm{~mm}$ diameter is larger than that of any tip with another diameter. Thus, $0.1 \mathrm{~mm}$ would be the best choice for the tip diameter.

The last crucial factor to be considered is the $Q$ factor. Lower $Q$ factors will result in lower stability for both FM-AFM and amplitude modulation AFM (AM-AFM) [17]. As shown in Figure 9, the $Q$ factor of the qPlus sensor is higher for shorter 


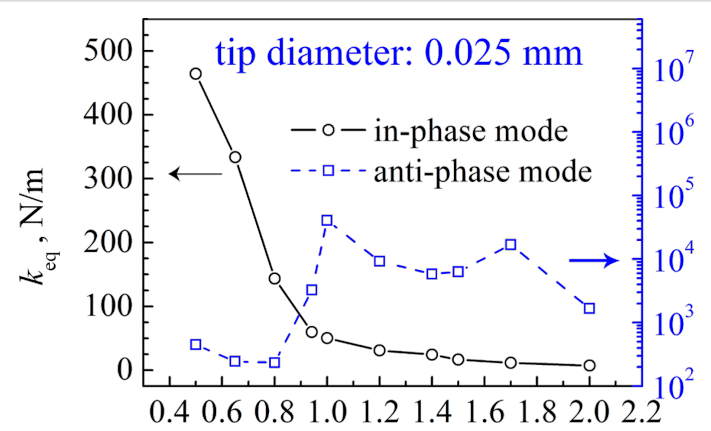

(a)

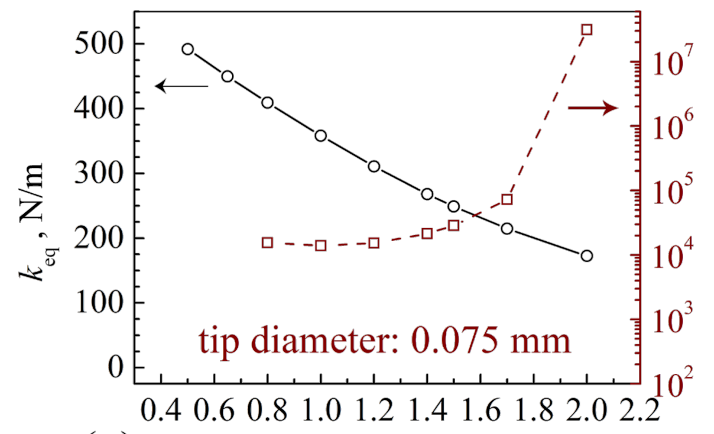

(c)

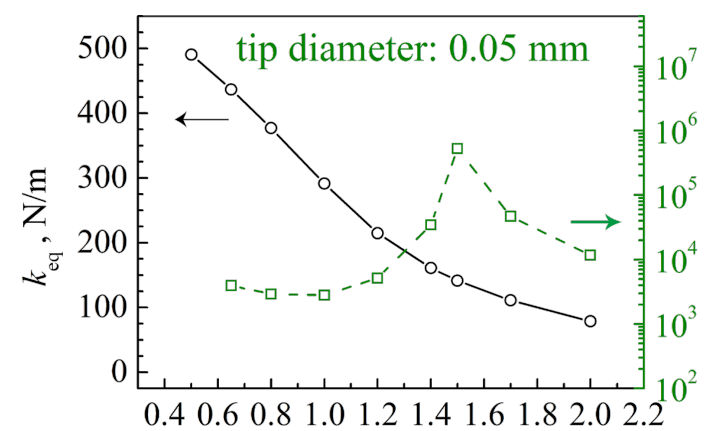

Length of tip, $\mathrm{mm}$

(b)

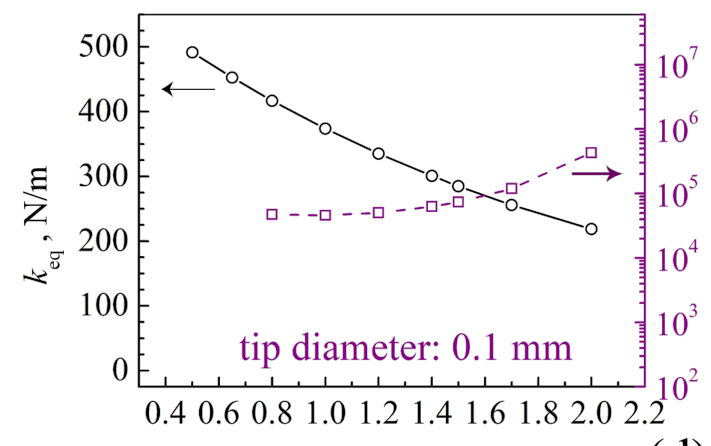

Length of tip, $\mathrm{mm}$

(d)

Figure 8: Equivalent stiffness $k_{\text {eq }}$ of the qPlus sensor as a function of the tip length depicted for the four different tip diameters.
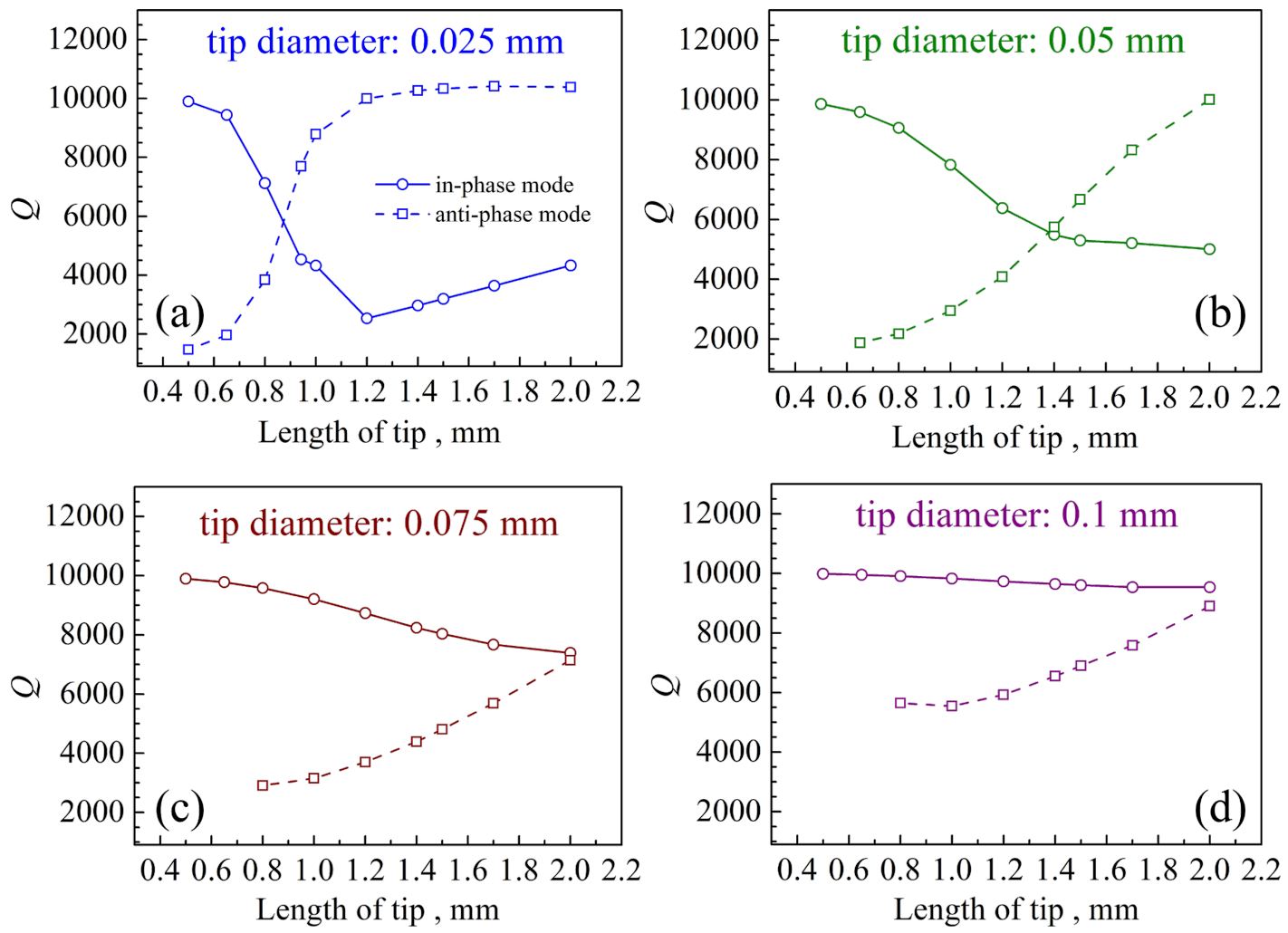

Figure 9: $Q$ factor as a function of the tip length depicted for the four different tip diameters. 
tips in the in-phase mode. The $Q$ factor of the $0.1 \mathrm{~mm}$ tip in the in-phase mode decreases the least when the length increases, and $A_{\text {tip }}$ is smallest when the tip length is shorter than $1.2 \mathrm{~mm}$ Therefore, in terms of resolution, a $0.1 \mathrm{~mm}$ tip with a length less than $1.2 \mathrm{~mm}$ in the in-phase mode would exhibit a better performance.

\section{Conclusion}

The oscillation characteristics of qPlus sensors with different dimensions of tungsten tips were studied by using the finite element method. The results reveal that the changes of the output current and the tuning fork amplitude $\left(A_{\mathrm{tfz}}\right)$ with respect to the tip length are not necessarily synchronous. The tip amplitude $\left(A_{\text {tip }}\right)$ is determined by vibrations of both the tuning fork prong and the tip. A $0.1 \mathrm{~mm}$ tip with a tip length smaller than $1.2 \mathrm{~mm}$ used in the in-phase mode can improve the spatial resolution. This research provides quantitative data to optimize the tip dimensions and the corresponding vibration modes of a qPlus sensor with a long tilted tip.

\section{Supporting Information}

\section{Supporting Information File 1}

Additional simulation results.

[https://www.beilstein-journals.org/bjnano/content/

supplementary/2190-4286-12-7-S1.pdf]

\section{Funding}

The authors gratefully acknowledge the support from the National Key Technologies R\&D Program of China (Grant No. 2016YFA0201101), the National Natural Science Foundation of China (Grants No. 61734008, and No. 11804369), and CAS Pioneer Hundred Talents Program (Song, W.).

\section{ORCID ${ }^{\circledR}$ iDs}

Kebei Chen - https://orcid.org/0000-0002-6873-8162 Gengzhao Xu - https://orcid.org/0000-0003-4121-1667

\section{References}

1. Giessibl, F. J. Principles and Applications of the qPlus Sensor. In Noncontact Atomic Force Microscopy: Volume 2; Morita, S.; Giessibl, F. J.; Wiesendanger, R., Eds.; Springer: Berlin, 2009; pp 122-132. doi:10.1007/978-3-642-01495-6

2. Giessibl, F. J. Rev. Sci. Instrum. 2019, 90, 011101. doi:10.1063/1.5052264

3. Giessibl, F. J. Appl. Phys. Lett. 1998, 73, 3956-3958. doi:10.1063/1.122948

4. Hembacher, S.; Giessibl, F. J.; Mannhart, J.; Quate, C. F. Proc. Natl. Acad. Sci. U. S. A. 2003, 100, 12539-12542. doi:10.1073/pnas.2134173100
5. Emmrich, M.; Huber, F.; Pielmeier, F.; Welker, J.; Hofmann, T.; Schneiderbauer, M.; Meuer, D.; Polesya, S.; Mankovsky, S.; Ködderitzsch, D.; Ebert, H.; Giessibl, F. J. Science 2015, 348, 308-311. doi:10.1126/science.aaa5329

6. Gross, L.; Mohn, F.; Liljeroth, P.; Repp, J.; Giessibl, F. J.; Meyer, G. Science 2009, 324, 1428-1431. doi:10.1126/science.1172273

7. Giessibl, F. J. Appl. Phys. Lett. 2000, 76, 1470-1472. doi:10.1063/1.126067

8. Wastl, D. S.; Weymouth, A. J.; Giessibl, F. J. ACS Nano 2014, 8, 5233-5239. doi:10.1021/nn501696q

9. Schneiderbauer, M.; Wastl, D.; Giessibl, F. J. Beilstein J. Nanotechnol. 2012, 3, 174-178. doi:10.3762/bjnano.3.18

10. Pielmeier, F.; Giessibl, F. J. Phys. Rev. Lett. 2013, 110, 266101. doi:10.1103/physrevlett.110.266101

11. Huff, T. R.; Dienel, T.; Rashidi, M.; Achal, R.; Livadaru, L.; Croshaw, J.; Wolkow, R. A. ACS Nano 2019, 13, 10566-10575. doi:10.1021/acsnano.9b04653

12. Pawlak, R.; Glatzel, T.; Pichot, V.; Schmidlin, L.; Kawai, S.; Fremy, S.; Spitzer, D.; Meyer, E. Nano Lett. 2013, 13, 5803-5807. doi:10.1021/nl402243s

13. Koopman, M.; de Bakker, B. I.; Garcia-Parajo, M. F.; van Hulst, N. F. Appl. Phys. Lett. 2003, 83, 5083-5085. doi:10.1063/1.1634385

14. Rensen, W. H. J.; van Hulst, N. F.; Kämmer, S. B. Appl. Phys. Lett. 2000, 77, 1557-1559. doi:10.1063/1.1308058

15. Pürckhauer, K.; Weymouth, A. J.; Pfeffer, K.; Kullmann, L.; Mulvihill, E.; Krahn, M. P.; Müller, D. J.; Giessibl, F. J. Sci. Rep. 2018, 8, 9330. doi:10.1038/s41598-018-27608-6

16. Ichii, T.; Fujimura, M.; Negami, M.; Murase, K.; Sugimura, H. Jpn. J. Appl. Phys. 2012, 51, 08KB08. doi:10.1143/jjap.51.08kb08

17. Higuchi, S.; Kuramochi, H.; Kubo, O.; Masuda, S.; Shingaya, Y.; Aono, M.; Nakayama, T. Rev. Sci. Instrum. 2011, 82, 043701. doi:10.1063/1.3569765

18. Kim, S.; Yoo, H.; Lee, K.; Friedman, B.; Gaspar, M. A.; Levicky, R. Appl. Phys. Lett. 2005, 86, 153506. doi:10.1063/1.1904713

19. Kwon, S.; Jeong, S.; Kang, Y. Rev. Sci. Instrum. 2011, 82, 043707. doi:10.1063/1.3582662

20. Cinar, E.; Sahin, F.; Yablon, D. Beilstein J. Nanotechnol. 2015, 6, 2015-2027. doi:10.3762/bjnano.6.205

21. Higuchi, S.; Kubo, O.; Kuramochi, H.; Aono, M.; Nakayama, T. Nanotechnology 2011, 22, 285205. doi:10.1088/0957-4484/22/28/285205

22. Sapozhnikov, I.; Gorbenko, O.; Felshtyn, M.; Zhukov, M.; Golubok, A. AIP Conf. Proc. 2019, 2064, 020003. doi:10.1063/1.5087659

23. Guo, T.; Wang, S.; Dorantes-Gonzalez, D. J.; Chen, J.; Fu, X.; Hu, X. Sensors 2011, 12, 175-188. doi:10.3390/s120100175

24. Thermo Fisher Scientific Ltd. Tungsten wire MSDS. https://chemicals.thermofisher.cn (accessed July 9, 2018).

25. van Vörden, D.; Lange, M.; Schmuck, M.; Schmidt, N.; Möller, R. Beilstein J. Nanotechnol. 2012, 3, 809-816. doi:10.3762/bjnano.3.90

26. Dagdeviren, O. E.; Schwarz, U. D. Beilstein J. Nanotechnol. 2017, 8, 657-666. doi:10.3762/bjnano.8.70

27. Thomson, W. T.; Dahleh, M. D. Euler Equation for Beams. In Theory of Vibration with Applications; Stenquist, W., Ed.; Prentice Hall: Upper Saddle River, U.S., 1998; pp 271-273.

28. Melcher, J.; Hu, S.; Raman, A. Appl. Phys. Lett. 2007, 91, 053101. doi:10.1063/1.2767173 


\section{License and Terms}

This is an Open Access article under the terms of the Creative Commons Attribution License (https://creativecommons.org/licenses/by/4.0). Please note that the reuse, redistribution and reproduction in particular requires that the author(s) and source are credited and that individual graphics may be subject to special legal provisions.

The license is subject to the Beilstein Journal of Nanotechnology terms and conditions: (https://www.beilstein-journals.org/bjnano/terms)

The definitive version of this article is the electronic one which can be found at:

$\underline{\text { https://doi.org/10.3762/bjnano.12.7 }}$ 